\title{
DEVELOPMENT AND TEST OF A FRINGE-IMAGING DIRECT- DETECTION DOPPLER WIND LIDAR FOR AERONAUTICS
}

\author{
Patrick Vrancken*, Jonas Herbst* \\ *Institute of Atmospheric Physics, German Aerospace Center (DLR), 82234 Weßling, Germany \\ Email: patrick.vrancken@dlr.de
}

\begin{abstract}
DLR currently investigates the use of Doppler wind lidar as sensor within feedforward gust alleviation control loops on fast-flying fixed-wing aircraft. Such a scheme imposes strong requirements on the lidar system such as sub-m/s precision, high rate, high spatial resolution, close measurement ranges and sensitivity to mixed and pure molecular backscatter.
\end{abstract}

We report on the development of a novel directdetection Doppler wind lidar (DD-DWL) within these requirements. This DD-DWL is based on fringe-imaging of the Doppler-shifted backscatter of UV laser pulses in a field-widened Michelson interferometer using a fast linear photodetector.

A prototype for airborne operation has been ground-tested in early 2018 against a commercial coherent DWL, demonstrating its ability of measuring close-range wind speeds with a precision of $0.5 \mathrm{~m} / \mathrm{s}$, independent of the actual wind speed.

\section{INTRODUCTION}

Applications of Doppler wind lidars (DWL) in aeronautics have been under discussion since their invention [1], and test flights of actually all generations of coherent DWLs (from $\mathrm{CO}_{2}$ to solid-state and fiber laser technology) and also direct-detection DWLs to detect turbulence and wake vortices have been undertaken since the early 70ies. Despite these efforts and despite turbulence being a major cause for (non-fatal) incidents in civil aeronautics, with increasing trend, none of these setups has found its way into an application or product so far. Apart from the objective complexity of lidars, possible reasons might be identified in the increasing structural resilience of aircraft towards the effects of turbulence. Today's urge for efficiency within the air transport sector ultimately implying more lightweight, and thus vulnerable structures, will

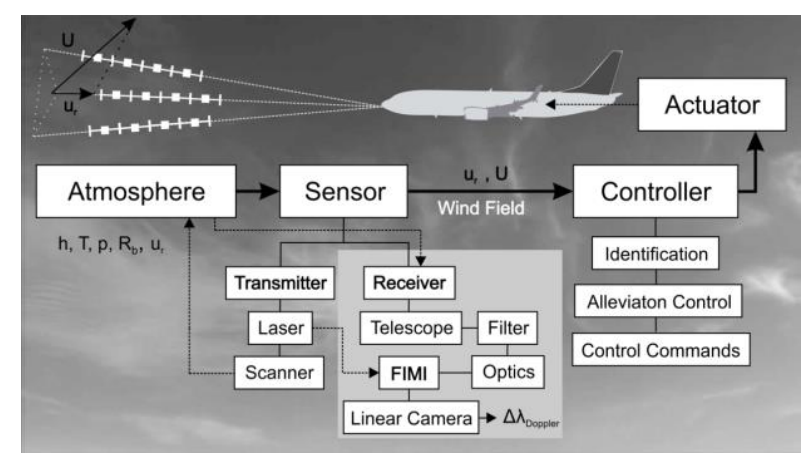

Figure 1: Lidar-based feed-forward gust alleviation system (the grey-shaded box is treated herein)

meet higher turbulence abundance in projected flight altitudes [2]. This shall lead to a turning point of the above described situation.

In this context, DLR-FT (Institute of Flight Systems) is studying feed-forward flight control systems for mitigation of atmospheric effects. Key ingredient for such feed-forward alleviation (for turbulent gusts or wake vortices) systems is a number of measured wind speed values in the flightpath ahead of the aircraft. Today's generation of these controllers may handle partial or comparatively low data yield (i.e. realistic in terms of lidar delivery) due to the use of a-priori information on the phenomenon to be encountered $[3,4]$. This dramatically decreases the requirements on DWLs for delivery of these wind data, i.e. pushing these into technically satisfiable bounds. These requirements are being iterated within the present studies, together with feasible lidar parameters, delivered by the LIDAR group of DLR-PA (Institute of Atmospheric Physics).

Briefly, owed to the high speed of high-flying aircraft (30 kft to $40 \mathrm{kft}$ ), such a feed-forward flight controller would impose following rough parameters on a DWL system: a full-field (several measurement directions, as within a cone, e.g.) update rate of 10 to $20 \mathrm{~Hz}$; a wind speed precision of around or less $1 \mathrm{~m} / \mathrm{s}$, mainly due to the angular penalty (line-of-sight / LOS measurement versus vertical required) in a cone-like scanning setup; 


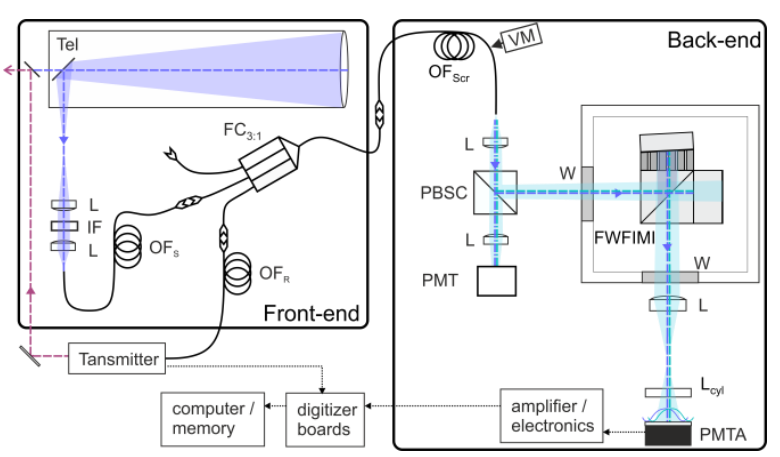

Figure 2: Simplified synopsis of FWFIMI-based Doppler Wind Lidar, technical details in the text

a spatial resolution of $20 \mathrm{~m}$ to $50 \mathrm{~m}$, depending on the aircraft mass, wingspan and flight speed (and thus its frequency response to turbulence); close ranges $50 \mathrm{~m}$ to $300 \mathrm{~m}$ ahead of the aircraft being comfortable with the SNR but actually representing challenges of optical nature; and last, full availability of the measurement in cruise flight conditions, dictated by certifying authorities, and eventually negating the use of aerosoldependent coherent DWLs.

DLR-PA theoretically studied and compared several possible implementation forms of directdetection (DD) DWLs [5]. We deliberately discarded coherent DWL for the risk of nonavailability due to possible virtual aerosol absence within the targeted clear air volumes of cruise flight airspace, following the same argumentation as the AWIATOR project before [6,7].

The needed close measurement range $(<300 \mathrm{~m})$ infers a maximized overlap within this region and thus a large field-of-view (FOV) of several mrad. Even for reasonable (i.e. aircraft-compatible) telescope sizes and $\sim$ back-end optics diameters, the magnification translates this FOV to important angular distributions. The here [5] studied interferometers within DD-DWLs thus have to be field-widened in order to accept such angular distributions without considerable loss of contrast. This field-widening (FW) may be realized with two-path interferometers such as Mach-Zehnder (MZ) and Michelson (MI), as opposed to multiple-path interferometers such as Fizeau and Fabry-Perot interferometers. Several groups have successfully adopted the MZ technique, as within LNG of French LATMOS [8] or the different generations of Ball Aerospace's OAWL lidar $[9,10]$, both using quadrature phase retrieval. Our group has implemented the fringe-imaging (FI) option of MI [5], for several reasons: First, a FIMI may be implemented with slanted mirrors, producing linear fringe patterns, easily to be imaged on fast linear detector arrays. This grants both optimum photon efficiency and possible high range resolution, in contrast to two-dimensional CCD-type detectors, as used in AWIATOR [6,7], e.g. Second, an airborne application calls for resilience towards vibrations and temperature variations, what may be responded to by a monolithic interferometer architecture, comparatively easily to be achieved with a MI. Third, a monolithic FIMI can be constructed to be both field widened (FWFIMI) and temperature compensated. Finally, such a FW design may additionally be arranged to be tilted to the incident light, enabling two-channel (transmit and reflected channels) operation.

Once the FW option established, this allows for implementing large-core multi-mode optical fibers (OF) for the transport of the backscattered optical flux from telescope collection to the interferometer, representing additional advantages: First, using OF eases the construction and implementation of such lidar systems, eventually for the operation within the confined space of an aircraft. They also allow for easy combination, splitting etc. via typical fiber modules such as couplers. Second, large-core OF provide scrambling [11, e.g.], i.e. annihilating of angular information, thus (at least theoretically) overcoming the need for range-resolved calibration. Further, OF allow for shaping the optical flux to nearly arbitrary shapes (like rectangular-top hat) better suited for detector illumination than "natural" circular-Gaussian ones. And last, while at the same time generating speckles, large-core OF facilitate the spatial averaging of atmospheric (molecular and aerosol backscatter-generated) speckles.

Our theoretical studies and simulations [5] regarding the possible performance of such an FWFIMI-based DD-DWL also addressed different types of pulsed laser types (regarding power and pulse repetition frequency - PRF), all in UV for its higher molecular backscatter and less eyesafety constraints. While these studies revealed the superiority of medium-high PRFs (around $1 \mathrm{kHz}$ ), in our demonstration experiments we used 
the airborne-proven WALES/DELICAT laser [12] with $100 \mathrm{~Hz}$ PRF due to its immediate availability. We realized the described FWFIMI option as an experimental DWL receiver system. In the next section we give a simplified synopsis of the developed FWFIMI-DD-DWL, while in Sect. 3 we shortly address the first ground-based validation experiments and their results.

\section{THE FWFIMI-DWL SYSTEM}

Fig. 2 shows a fairly simplified schematic of the DW lidar setup. The frequency-locked laser transmitter emits short UV laser pulses into the atmosphere in a mono-static, co-axial arrangement with the receiver telescope. The lidar receiver may be broken down into a front-end (RFE) and backend (RBE) part. The RFE has the simple function of collecting the backscattered light, collimating it (by lens L, see Fig. 2) for background noise suppression by a narrow interference filter (IF) and subsequent concentration (L) in a large-core $(600 \mathrm{~m})$ optical fiber $\left(\mathrm{OF}_{\mathrm{S}}\right.$, with subscript $\mathrm{S}$ for signal) which actually defines the FOV. The transmitter is also equipped with a fiber launch and thus delivers its spectral reference pulses in an $\mathrm{OF}_{\mathrm{R}}$ (subscript $\mathrm{R}$ for reference) that further serves as delay line in order to arrange the reference laser pulses temporally after the Doppler-shifted signal. Both paths are combined within a 3:1 custombuilt $600 \mathrm{~m}$ fiber coupler $\left(\mathrm{FC}_{3: 1}\right)$. The third port may be used for analysis illumination by a UV LED, e.g.

For transport of the light to the RBE, scrambling and beamforming, we implemented, after performance tests of various large-core multimode $\mathrm{OF}$, a square-core $\mathrm{OF}_{\text {Scr }}$ of $600 \mathrm{~m}$ edge length, including a vibrating device (VM) for temporal fiber speckle averaging.

The RBE comprises the collimation of the light from the $\mathrm{OF}_{\text {Scr }}$ (by lens $\mathrm{L}$ ), and the polarization separation by a polarizing beam splitter cube (PBSC). The loss of polarization (and thus $50 \%$ of the signal power here) is the major disadvantage of using MM OF since the beamsplitter coating of the MI requires s-polarization for delivering adequate fringe contrast. The p-part is focused onto a single PMT used for calibration and alignment (overlap) purposes. The s-part is then

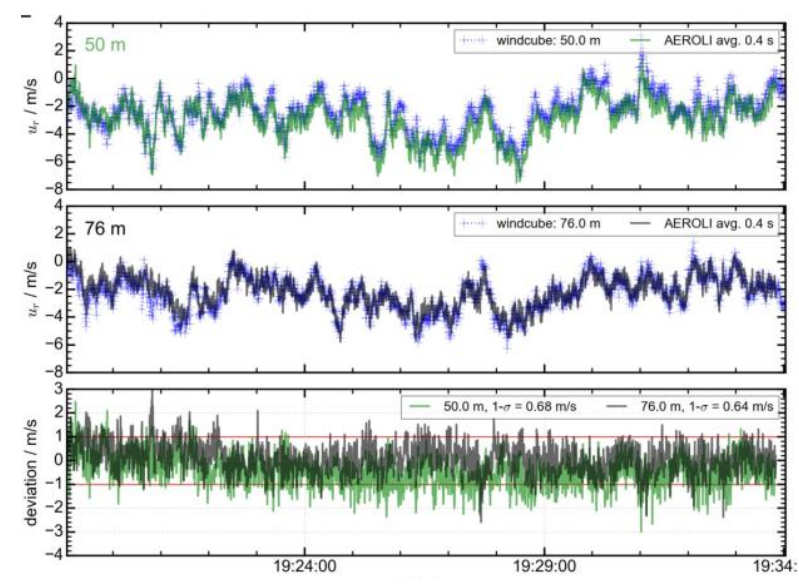

Figure 3: Exemplary horizontal wind measurement time series of the FWFIMI-DWL, bias removed, compared to Windcube ${ }^{\circledR} 200 S$ data, both averaged to $2.5 \mathrm{~Hz}$ rate at center ranges 50 and $76 \mathrm{~m}$. Lower panel: Respective deviations between the DWLs

fed into the MI which is mounted in an Invar bracket within a steel temperature-controlled housing (with optical windows W). The FWFIMI consists of a cubic beamsplitter of $30 \mathrm{~mm}$ edge length, an air arm of $11 \mathrm{~mm}$ with composite spacers (for temperature tuning optimization) and skewed mirror, and a $16 \mathrm{~mm}$ glass arm; these elements are all optically-contacted one to another resulting in a compact monolithic device.

The fringe localization plane is imaged by a lens L onto 16 elements of a linear photomultiplier array (PMTA), the vertical dimension (orthogonal to sketch plane in Fig. 2) being compressed by a cylindrical lens $\left(\mathrm{L}_{\mathrm{cyl}}\right)$. The signal current of each element of the PMTA is converted to a voltage by a transimpedance amplifier and is then digitized by two eightchannel 16 bit, $30 \mathrm{MHz}-$ bandwidth analog-todigital converter boards, yielding a sampling rate of $30 \mathrm{MS} / \mathrm{s}$. The atmospheric signal, the laser reference signal and a pre-trigger background signal are stored on a computer for each shot.

Currently, the retrieval of wind speed is performed offline since this routine is subject of ongoing research. The imaged compressed fringe amplitude has the approximate form of a cosine with skewness and kurtosis, resulting from imperfections of the MI optical surfaces, illumination etc. This fringe has to be corrected by an illumination function which is determined by a laser frequency sweep over the MI free spectral range (FSR). After a certain range averaging (six 
bins in the following example) and temporal averaging ( 40 pulses here) the temporally separated signal and reference fringes are each approximated by a four-parameter function (including the phase $\phi$ ) employing a downhill search method. The phase comparison directly yields the wind speed, a shift of $5.6 \mathrm{MHz}$ $(\approx 1 / 1900$ of the FSR of $10.7 \mathrm{GHz}$ ) translating to $1 \mathrm{~m} / \mathrm{s}$.

\section{GROUND-BASED VALIDATION}

For validation of the concept and of our physical implementation we performed ground-based tests at DLR Oberpfaffenhofen. Here, our FWFIMIDWL was arranged with horizontal LOS, alongside a commercial coherent DWL (Leosphere Windcube ${ }^{\circledR}$ 200S), the latter set at most to similar settings in terms of focusing, range and time averaging. Fig. 3 shows such comparative measurements for synthetic range gates of $30 \mathrm{~m}$, centered at $50 \mathrm{~m}$ and $76 \mathrm{~m}$ distance to the lidar over a $15 \mathrm{~min}$ interval. An important bias (see below) has been removed but the respective short-term fluctuations of the very moderate wind fit well one to another. The lower panel shows the (bias-removed) difference between the two DWL series. Here, a joint standard deviation of $0.7 \mathrm{~m} / \mathrm{s}$ may be determined. Assuming an upper bound for the Windcube ${ }^{\circledR}$ precision of $0.5 \mathrm{~m} / \mathrm{s}$ (specified by the manufacturer), our DWL also yields this level of precision. Pearson correlation coefficients of 0.89 and 0.86 , respectively, have been determined for these two series.

The above mentioned illumination function currently is a key point of investigation since its spurious fluctuations ultimately lead to timevariable bias of the wind measurement.

The shown analysis does not yet include investigations regarding signal-to-noise ratio and many other intricacies but merely demonstrates the potential of such a FWFIMI-DWL setup.

\section{CONCLUSION AND OUTLOOK}

We demonstrated the to our knowledge first direct-detection Doppler wind lidar based on fringe-imaging within a field-widened Michelson interferometer. We could show theoretically, by simulation and experimentally that this architecture is fundamentally appropriate for delivering wind speed measurements at high spatial and temporal resolution while yielding sub-m/s precision. In the near future we strive to evolve and enhance our experimental system based on the deficiencies identified so far. Part of the activities ahead are atmospheric trials in high altitude (i.e. molecular backscatter conditions), ultimately aiming airborne tests.

\section{REFERENCES}

[1] P. Vrancken, in Aviation Turbulence, edited by R. Sharman and T. Lane (Springer Int. 2016).

[2] P. D. Williams, Advances in Atmospheric Sciences 34, 576 (2017).

[3] J. Ehlers et al., in 14th AIAA Aviation Technology, Integration, and Operations Conference (AIAA 2014).

[4] N. Fezans and H.-D. Joos, in AIAA Atmospheric Flight Mechanics Conference (AIAA 2017).

[5] J. Herbst and P. Vrancken, Applied Optics 55, 6910 (2016).

[6] N. P. Schmitt et al., Aerospace Science and Technology 11, 546 (2007).

[7] G. J. Rabadan et al., Journal of Aircraft 47, 392 (2010).

[8] D. Bruneau et al., Appl. Opt., AO 54, 8776 (2015).

[9] S. C. Tucker et al., in Lidar Remote Sensing for Environmental Monitoring XV (SPIE 2015), p. $96120 \mathrm{E}$.

[10] S. C. Tucker et al., JTECH 35, 2079 (2018).

[11] G. Avila et al., in Proc. SPIE 7735, Ground-based and Airborne Instrumentation for Astronomy III, (2010), p. 773588.

[12] P. Vrancken et al., Applied Optics 55, 9314 (2016). 